\title{
Comparison of long-term treatment outcomes of T2N0M0 laryngeal squamous cell carcinoma using different treatment methods
}

\author{
EUN KYUNG JUNG ${ }^{1}$, SEONG-MIN JIN ${ }^{1}$, JAE-GU KIM ${ }^{1}$, JAE-UK JUNG ${ }^{2}$, DONG HOON LEE ${ }^{1}$, \\ JOON KYOO LEE ${ }^{1}$, SANG CHUL LIM ${ }^{1}$, WOONG-KI CHUNG ${ }^{2}$, HEE KYUNG KIM ${ }^{3}$, JUN-EUL HWANG ${ }^{3}$, \\ HYUN-JEONG SHIM ${ }^{3}$, WOO-KYUN BAE ${ }^{3}$, SANG-HEE CHO ${ }^{3}$, IK-JOO CHUNG ${ }^{3}$ and TAE MI YOON ${ }^{1}$ \\ Departments of ${ }^{1}$ Otorhinolaryngology-Head and Neck Surgery; ${ }^{2}$ Radiation Oncology and ${ }^{3}$ Internal Medicine, \\ Chonnam National University Medical School and Hwasun Hospital, \\ Hwasun, Jeollanam 519-763, Republic of Korea
}

Received September 19, 2019; Accepted April 1, 2020

DOI: 10.3892/ol.2020.11628

\begin{abstract}
Early [stage I and II (T2N0M0)] laryngeal cancer types are currently recommended to be treated with a single modality, consisting of definitive radiation therapy or larynx-preserving surgery. Although the treatment outcomes of stage I are good, the frequency of successful outcomes decreases with T2N0M0. Therefore, the present study investigated the treatment outcomes of different treatment methods in T2NOM0 laryngeal cancer. In total, 83 patients with previously untreated T2NOM0 laryngeal squamous cell carcinoma were enrolled. Patients were grouped by treatment method: Radiation therapy (RT; 27 patients); chemoradiotherapy (CRT; 46 patients) with cisplatin base; and surgery-based therapy (SBT; ten patients). The recurrence rates of the RT, CRT and SBT groups were 44.4, 19.6 and 50\%, respectively. Moreover, the local control rates of the RT, CRT and SBT groups were 55.6, 87.0 and $80 \%$, respectively. The CRT group had a significantly lower recurrence rate and higher local control rate compared with the $\mathrm{RT}$ group $(\mathrm{P}<0.05)$. In the survival analysis, overall and disease-specific survival rate did not differ significantly among the treatment groups. However, 3- and 5-year disease-free survival rates (DFS) of the RT group were both 55\%, those of the SBT group were both $50 \%$ and those of the CRT group were both $80 \%$. Furthermore, the DFS was significantly higher in CRT group compared with the other groups $(\mathrm{P}=0.02)$. Using multivariate analysis with Cox regression, it was found that the treatment method was
\end{abstract}

Correspondence to: $\mathrm{Dr}$ Tae $\mathrm{Mi}$ Yoon, Department of Otorhinolaryngology-Head and Neck Surgery, Chonnam National University Medical School and Hwasun Hospital, 322 Seoyang-Ro, Hwasun, Jeollanam 519-763, Republic of Korea

E-mail: yoontm@chonnam.ac.kr

Key words: laryngeal neoplasms, chemoradiotherapy, radiotherapy, survival rate, disease-free survival the most important factor for DFS and had a significant impact in the CRT group. In addition, in patients with glottic cancer with anterior commissure and subglottic invasion, the CRT group had significantly improved DFS compared with the RT group, whereas there was no significant difference between the two groups in patients without subglottic invasion. According to National Cancer Institution Common Toxicity Criteria (version 5.0), more patients had toxicity in the CRT group compared with the RT group. However, in the RT and CRT groups, no patients demonstrated mortality due to toxicity, and treatment-related toxicities were manageable. Collectively, although definitive conclusions could not be established, due to the limitations of this retrospective study, the results suggest that CRT had a positive impact on the local control and DFS rates with manageable toxicity in patients with T2N0M0 laryngeal cancer.

\section{Introduction}

Laryngeal cancer remains one of the most frequently detected head and neck cancer types (1); 40\% of affected patients are diagnosed in the early stage, mostly due to seeking medical treatment for hoarseness $(2,3)$. According to the most recent National Comprehensive Cancer Network guidelines (NCCN), early laryngeal cancers of stage I (T1N0M0) and stage II (T2N0M0) should be treated with definitive radiation therapy (RT) or surgery, such as laryngeal laser surgery or partial laryngectomy (4). It has been reported that treatment outcomes in terms of local control, laryngeal preservation and survival rates are similar between those methods in patients with T1NOM0 and T2NOM0 laryngeal cancer (3-6).

The local control rate after RT alone is reportedly $80-95 \%$ in patients with T1N0M0 laryngeal cancer (5-7); however, the results change in T2N0M0 disease. Treatment outcomes of T2N0M0 laryngeal cancer via RT alone and organ-preserving surgery, with 50-85\% local control rates, are insufficient compared with those of T1N0M0 laryngeal cancer (7-11). Among the various attempts to improve the treatment outcomes of patients with T2N0M0 laryngeal cancer, previous studies 
have suggested combining chemotherapy with RT $(7,12,13)$. Moreover, chemoradiation therapy (CRT) has been revealed to increase the radiosensitivity of cancer cells, thus maximizing the local control effect (14-16). In stage III and IV laryngeal cancer, the comparative advantage of CRT has been reported in several randomized trials $(17,18)$. In T2N0M0 laryngeal cancer, however, no randomized trials have compared treatment outcomes among RT alone, CRT or surgery-based treatment (SBT), and retrospective studies have been limited.

Therefore, the present study compared the long-term treatment outcomes, including overall survival (OS) rates, disease-specific survival (DSS) rates, local control rates and disease-free survival (DFS) rates, of patients with T2N0M0 laryngeal cancer treated with RT, CRT or SBT. Furthermore, this study aimed to provide an important basis for identifying the impact of CRT on improving the prognosis of patients with T2N0M0 laryngeal cancer.

\section{Patients and methods}

Patient selection. Between March 2005 and July 2015, consecutive patients with previously untreated T2N0M0 laryngeal squamous carcinoma, who were treated in Chonnam National University Hwasun Hospital (Hwasun, South Korea) were enrolled in the present study. This study was conducted in a tertiary referral hospital with a regional cancer center in South Korea. TNM staging, according to the 8th American Joint Committee on Cancer staging system was used (19). In all patients, flexible laryngoscopic examination, diagnostic biopsy, computed tomography (CT) and ${ }^{18} \mathrm{~F}-\mathrm{FDG}$ positron emission tomography (PET)-CT were performed for accurate staging before the treatment, and these imaging examinations were reviewed to prevent inappropriate enrollment in this study. Patients for whom a full record of their staging, treatment method and follow-up results, including radiologic studies and physical examinations, was unavailable were excluded. After a retrospective review of the charts of 86 patients, three patients were excluded. In total, two patients did not complete RT, due to financial problems or treatment refusal, while one patient had double primary cancer (lung and larynx), which could have confounded the interpretation of the treatment outcomes. Thus, 83 patients (81 men and 2 women; age range, $47-85$ years; mean age, 65.8 years), were ultimately enrolled in this study.

Treatment methods. The enrolled patients were classified by treatment method into the RT-only $(n=27)$, CRT $(n=46)$ and SBT $(n=10)$ groups. The treatment decision for patients was determined by considering numerous factors, including lung and kidney function, the profession of the patient (voice demand), lifestyle requirements and psychological support, as well as the cancer extent $(20,21)$.

All patients in the RT-only and CRT groups underwent RT with curative intent. External beam RT was performed using three-dimensional RT (3D-CRT) or intensity modulated RT (IMRT). The patients received 3D-CRT with $6 \mathrm{MV}$ photon beams produced by a Clinac IX (Varian Medical Systems, Inc.) using the field-shrinking technique (22). IMRT was conducted using a Clinac IX or TomoTherapy (Accuray, Inc.). The gross target volume (GTV) was the macroscopic extent of laryngeal disease on CT, magnetic resonance imaging and PET-CT. The clinical target volume (CTV) was delineated as a 1-2 cm margin from GTV, with consideration of microscopic disease or larynx with a field of $6 \times 6 \mathrm{~cm}$. The radiation dose for CTV was administered at 2 Gy per dose, 66-70 Gy, five times a week. Elective nodal irradiation, including bilateral level II, III and IV, was performed with a dose of 45 Gy. Most patients, except three patients who underwent surgery only, received bilateral elective nodal irradiation. Patients in the CRT group underwent induction chemotherapy with either radiotherapy or concurrent CRT (CCRT), or CCRT only. Then, two cycles of induction chemotherapy were performed using cisplatin $\left(75 \mathrm{mg} / \mathrm{m}^{2}\right)$ and 5-fluorouracil (5-FU) $\left(1,000 \mathrm{mg} / \mathrm{m}^{2}\right)$ with/without docetaxel $\left(70 \mathrm{mg} / \mathrm{m}^{2}\right)$. Both followed CCRT, patients who had induction chemotherapy with CCRT, and CCRT only, patients who had CCRT only without induction chemotherapy, was performed with cisplatin-based treatment with RT. Dosage of chemotherapy was reduced to $75 \%$ of the original dose in subsequent cycles when patients had toxicity $>$ grade III hematologic or non-hematologic toxicity. After induction chemotherapy, flexible laryngoscopic exam and CT was performed to assess the response of chemotherapy. If some patients had progressive or stable disease, surgery was planned instead of RT or CCRT. However, none of the patients underwent surgery on poor response of induction chemotherapy.

SBT-grouped patients had surgery only or surgery with adjuvant RT. The adjuvant RT was performed as aforementioned. The radiation dose of adjuvant $\mathrm{RT}$ was administered at 50-68 Gy.

Patient characteristics. The characteristics of the 83 enrolled patients (men 81 ; women 2; mean age, 65.8 \pm 8.0 years; age range, 47-85 years) are shown in Table I. The mean patient follow-up period was $70.8 \pm 37.9$ months (follow-up range, 10.7-153.3 months). The distribution of the primary subsite was as follows: Glottis, 43 cases; supraglottis, 36 cases; and subglottis, four cases. In 43 patients with glottic cancer, 42 patients, except one patient with impaired vocal cord mobility, had the tumor extent to the supraglottis or subglottis. In 36 patients with supraglottic cancer, all patients had the tumor invade the mucosa of the adjacent subsite of supraglottis or glottis. In four patients with subglottic cancer, all patients had tumor extend to the vocal cords. With regards to the smoking history, 48 patients $(57.8 \%)$ were current smokers, 33 patients $(39.8 \%)$ were ex-smokers and two patients $(2.4 \%)$ did not have a smoking history. For alcohol history, 50 patients $(60.2 \%)$ were social drinkers, ten patients (12\%) were heavy drinkers and 23 patients $(27.7 \%)$ did not have an alcohol history. For primary treatment, 27 patients had RT alone, 46 patients had CRT and ten underwent SBT. Given that the distribution of primary sites was different for each treatment group, subgroup analysis was performed, according to the primary site, when analyzing the treatment outcome. In addition, analyses concerning anterior commissure and subglottic extension, which can effect glottic cancer treatment outcomes, were also performed.

Treatment response assessment and toxicity evaluation. Treatment response for the primary lesion and evaluation of lymph nodes and distant metastasis were assessed via physical 
examination involving flexible laryngoscope exam, CT and PET-CT in the fourth and twelfth weeks after the end of the first treatment. Regular follow-up was performed every 1-6 months thereafter.

Recurrence was defined as the first treatment failing due to the primary lesion or other sites including nodal or distant metastasis (7). Local control was defined as cure of the primary lesion without recurrence after the first treatment (7). Patients who experienced recurrence underwent salvage treatments such as surgery, chemotherapy or RT. Furthermore, treatment failure was defined as a disease with inoperable locoregional progression or distant metastasis despite the salvage treatment (7). The recurrence rate, local control rate and treatment failure rate were defined as an event occurring within the follow-up period without regular periods as 3- or 5-year.

OS was calculated from the date of the first diagnosis to mortality, or the last follow-up. DSS was calculated from the date of the first diagnosis to laryngeal cancer-associated mortality or the last follow-up. DFS was calculated from the date of diagnosis to the date of the first recurrence or the last follow-up.

Acute toxicity during RT and chemotherapy was graded according to the National Cancer Institution (NCI) Common Toxicity Criteria (CTC) version 5.0 (23). Laboratory results regarding hematologic, hepatic, renal and non-hematologic complications such as dermatitis, mucositis, nausea, diarrhea and fever, occurring after and during treatment were reviewed.

Statistical analysis. Survival curves were calculated by the Kaplan-Meier method and compared using the log-rank test, and the Renyi-type test from the survMisc Package in R. Multivariate analysis was performed using by Cox's regression. Unpaired t-test and Fisher's exact test were used to compare between two groups. ANOVA and nonparametric Kruskal-Wallis test was used for multiple comparison and the post hoc test was performed by Bonferroni. Analyses were performed using SPSS version 23.0 (SPSS, Inc.) and R version 3.9.2 ( $\mathrm{R}$ Foundation for Statistical Computing). $\mathrm{P}<0.05$ was considered to indicate a statistically significant difference.

\section{Result}

Local control, recurrence and treatment failure. For primary treatment, 27 patients had RT alone, 46 patients had CRT and ten underwent SBT. The difference in the distribution of the primary subsite was significant among the treatment groups (Table I; $\mathrm{P}=0.002$ ). Accordingly, when analyzing treatment outcomes, subgroup analysis by primary subsite was conducted. No significant differences were observed in age, sex or RT dose (Table I).

Recurrence was observed in 26/83 patients. Moreover, the RT, CRT and SBT groups had significantly different recurrence rates of $44.4 \%(12 / 27), 19.6 \%(9 / 46)$ and $50 \%(5 / 10)$ (Table II; $\mathrm{P}=0.03)$. In the subgroup analysis, the CRT group had a significantly lower recurrence rate compared with the RT group $(\mathrm{P}=0.03)$. Among the 26 patients, 12 were treated successfully. After salvage treatment, treatment failure was not associated with treatment group (Table II; $\mathrm{P}=0.24$ ).

Among the 27 patients in the RT group, 12 experienced recurrence in the primary site and four had distant metastasis.
Furthermore, the local control rate of the RT group was $55.6 \%(15 / 27)$. Among these 12 patients, five were successfully salvaged by surgery and adjuvant chemotherapy. However, after salvage treatment, seven patients $(25.9 \%)$ experienced treatment failure.

Among the 46 patients in the CRT group, six experienced recurrence in the primary site, four had nodal recurrence and three had distant metastasis. Moreover, the local control rate of the CRT group was $87.0 \%$ (40/46). The CRT group also had a significantly higher local control rate compared with the RT group. Among these nine patients, four were successfully salvaged by surgery, adjuvant chemotherapy and RT. After salvage treatment, five $(10.9 \%)$ experienced treatment failure.

Among the ten patients in the SBT group, two experienced recurrence in the primary site, one had nodal recurrence and two had distant metastasis. The local control rate of the SBT group was $80.0 \%$ (8/10). Among these five patients, three could be successfully salvaged by surgery, adjuvant chemotherapy and RT, while two (20\%) experienced treatment failure.

Survival rates. The 3- and 5-year OS of all patients were 79 and 69\%, respectively (Fig. 1A). Moreover, the 3- and 5-year DSS of all patients were 87 and $86 \%$, respectively (Fig. 1B). During a mean long-term follow-up duration of 70.8 months, 12 mortalities occurred due to disease progression and 16 due to causes unrelated to the laryngeal cancer, such as second primary cancer (lung cancer in two patients; esophageal cancer, hepatocellular carcinoma, cholangiocarcinoma, pancreatic cancer and myelodysplastic syndrome in one patient each); pneumonia in 5; and brain hemorrhage, esophageal variceal bleeding, urinary tract infection and unknown in one patient each. In addition, the 3- and 5-year DFS of all patients were both $68 \%$ (Fig. 1C).

The 3- and 5-year OS of the RT group were 74 and 65\%, those of the CRT group were 82 and $73 \%$, and those of the SBT group were 80 and $53 \%$, respectively $(\mathrm{P}=0.23$; Fig. $2 \mathrm{~A})$. The 3 - and 5-year DSS of the RT group were 85 and $80 \%$, while those of the CRT and SBT groups were both $89 \%(\mathrm{P}=0.29$; Fig. 2B).

However, the 3- and 5-year DFS in the RT group was both $55 \%$, those of the SBT group were both $50 \%$ and those of the CRT group were both $80 \%$. Moreover, the DFS demonstrated significant differences among treatment methods $(\mathrm{P}=0.02$; Fig. 2C). In the subgroup analysis, the DFS of the CRT group was significantly higher compared with those of the RT and SBT groups $(\mathrm{P}=0.02)$. To assess the significant factors for DFS, multivariate Cox regression analysis was performed of the following variables: Age, sex, treatment method and RT dose. Among the variables, the treatment method was the most important factor that influenced DFS, and CRT had a superior prognosis compared with RT and SBT (Table III).

Treatment outcomes of glottic cancer. In total, 43 patients with glottic cancer were enrolled in this study: 21 had RT alone, 20 had CRT and two had SBT. Of them, 35 (81.4\%) had anterior commissure $(\mathrm{AC})$ invasion, while 23 patients $(53.5 \%)$ had $\mathrm{AC}$ and subglottic invasion (data not shown). The SBT group was small, and thus the treatment outcomes of only the RT and CRT groups were compared. A total of 13 patients (30.2\%) experienced recurrence. Furthermore, the CRT group had a tendency 
Table I. Characteristics of patients.

\begin{tabular}{|c|c|c|c|c|c|}
\hline Parameters & Total $(\mathrm{n}=83)$ & RT alone $(\mathrm{n}=27)$ & CRT $(\mathrm{n}=46)$ & SBT $(n=10)$ & P-value \\
\hline Age, years & $65.8(47-85)$ & $67.5(47-85)$ & $64.5(51-77)$ & $66.9(53-76)$ & 0.292 \\
\hline Sex & & & & & 0.214 \\
\hline Male & 81 & 27 & 45 & 9 & \\
\hline Female & 2 & 0 & 1 & 1 & \\
\hline Primary subsite & & & & & 0.002 \\
\hline Glottis & 43 & 21 & 20 & 2 & \\
\hline Supraglottis & 36 & 6 & 22 & 8 & \\
\hline Subglottis & 4 & 0 & 4 & 0 & \\
\hline RT dose, Gy & $66.6(50.4-71.0)$ & $67.2(57.5-71.0)$ & $66.9(57.6-71.0)$ & $62.5(50.4-68.4)$ & 0.081 \\
\hline Follow-up duration, months & $70.8(10.7-153.3)$ & $72.2(14.1-149.9)$ & $72.6(10.7-145.6)$ & $58.8(17.1-153.3)$ & 0.573 \\
\hline
\end{tabular}

Data are presented as n or mean (range). RT, radiation therapy; CRT, chemoradiotherapy; SBT, surgery-based treatment.

Table II. Pattern of the recurrence and treatment failure according to the treatment method.

\begin{tabular}{|c|c|c|c|c|c|}
\hline Parameters & Total $(n=83)$ & $\mathrm{RT}(\mathrm{n}=27)$ & CRT $(n=46)$ & SBT $(n=10)$ & P-value \\
\hline Recurrence & & & & & 0.03 \\
\hline No & $57(68.7 \%)$ & $15(55.6 \%)$ & $37(80.4 \%)$ & $5(50 \%)$ & \\
\hline Yes & $26(31.3 \%)$ & $12(44.4 \%)$ & $9(19.6 \%)$ & $5(50 \%)$ & \\
\hline \multicolumn{6}{|l|}{ Recur sites } \\
\hline Primary recur & 20 & 12 & 6 & 2 & \\
\hline Nodal recur & 5 & 0 & 4 & 1 & \\
\hline Distant metastasis & 9 & 4 & 3 & 2 & \\
\hline Treatment failure & & & & & 0.24 \\
\hline No & $69(93.1 \%)$ & $20(74.1 \%)$ & $41(89.1 \%)$ & $8(80 \%)$ & \\
\hline Yes & $14(16.9 \%)$ & $7(25.9 \%)$ & $5(10.9 \%)$ & $2(20 \%)$ & \\
\hline
\end{tabular}

Recurrence happened on more than one single site in patients. Therefore, summation of recurred site does not concur with number of patients who recurred in each treatment group.

towards a lower recurrence rate compared with the RT group (42.9\% in RT vs. $15 \%$ in CRT); however, there was no statistical significance $(\mathrm{P}=0.09$; data not shown). It was indicated that the DFS of the CRT group was higher compared with the RT group (56\% 3- and 5-year DFS in RT vs. 85\% in CRT); however, this difference was not statistically significant $(\mathrm{P}=0.053)$. In addition, no significant differences were seen in OS and DSS according to RT and CRT groups ( $\mathrm{P}>0.05$; data not shown).

To evaluate the impact of AC and subglottic invasion on treatment outcomes of glottic cancer, a subgroup analysis was performed according to $\mathrm{AC}$ and subglottic invasion status. In 21 patients with AC and subglottic invasion, the DFS of the CRT group was significantly higher compared with the RT group ( $\mathrm{P}=0.045$; Fig. 3A). However, in 20 patients without subglottic invasion, DFS did not differ between the RT and CRT groups ( $\mathrm{P}=0.47$; Fig. $3 \mathrm{~B})$.

Treatment outcomes of supraglottic cancer. In total, 36 patients with supraglottic cancer were enrolled in this study: Six had RT alone, 22 had CRT and eight had SBT (Table I). Moreover,
12 patients (33.3\%) experienced recurrence (data not shown). The CRT group had a lower recurrence rate compared with the RT and SBT groups (50.0\% in RT and SBT vs. $22.7 \%$ in CRT, data not shown); however, there was no statistical significance $(\mathrm{P}=0.24$; data not shown). Furthermore, no significant differences were seen in OS, DSS and DFS according to treatment methods $(\mathrm{P}>0.05$; data not shown).

Toxicity. The RT and CRT groups were graded according to NCI-CTC version 5.0 (Table IV). It was found that more patients in the CRT group compared with the RT group showed symptoms of toxicity. Furthermore, the RT group had grade I dermatitis, followed by mucositis. Although the RT group demonstrated more non-hematologic toxicity, neutropenia was the most common finding in hematologic toxicity. However, neutropenia occurred with grade I toxicity, which was not life threatening. Moreover, there were no cases of grade IV toxicity among the RT group, and only four grade III events were identified. In addition, no treatment-associated mortalities occurred in the RT group. 

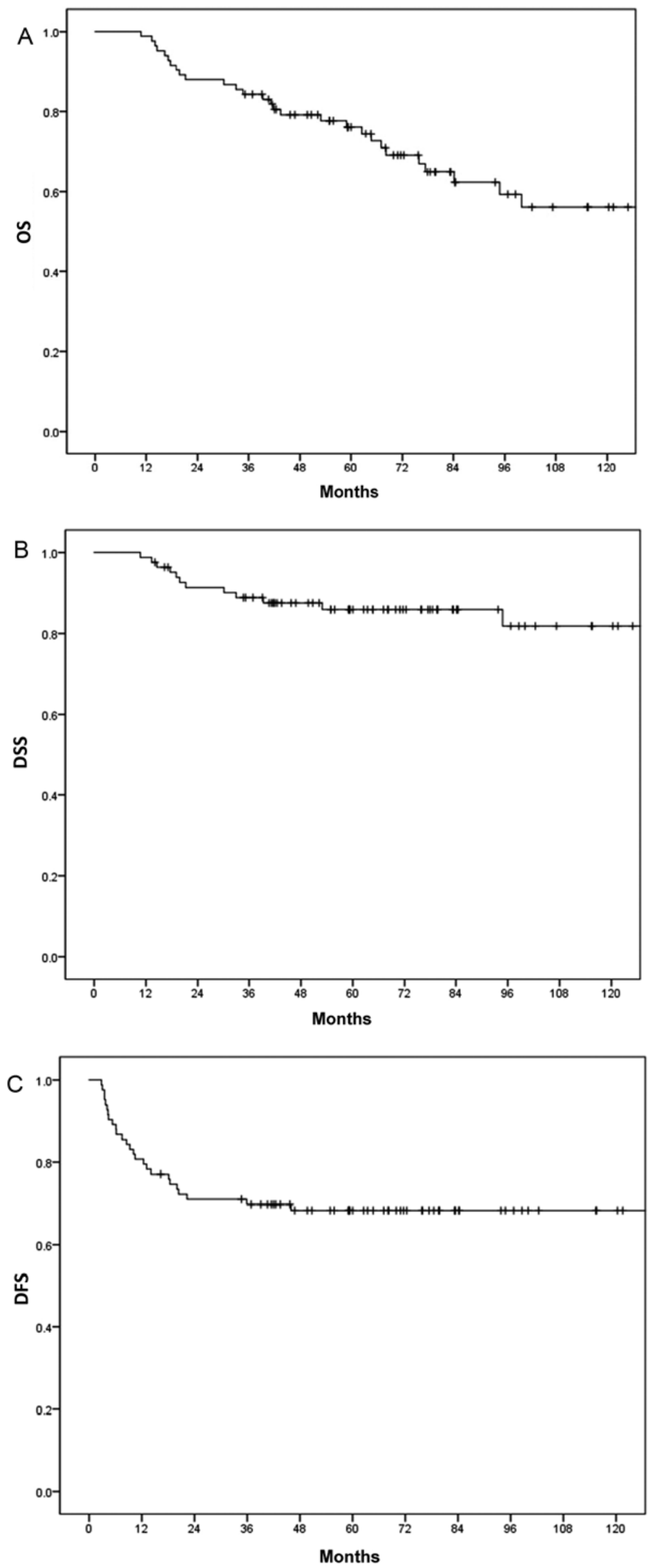

Figure 1. Survival rates. (A) OS, (B) DSS and (C) DFS of the 83 study patients. OS, overall survival; DSS, disease-specific survival; DFS, disease-free survival. 

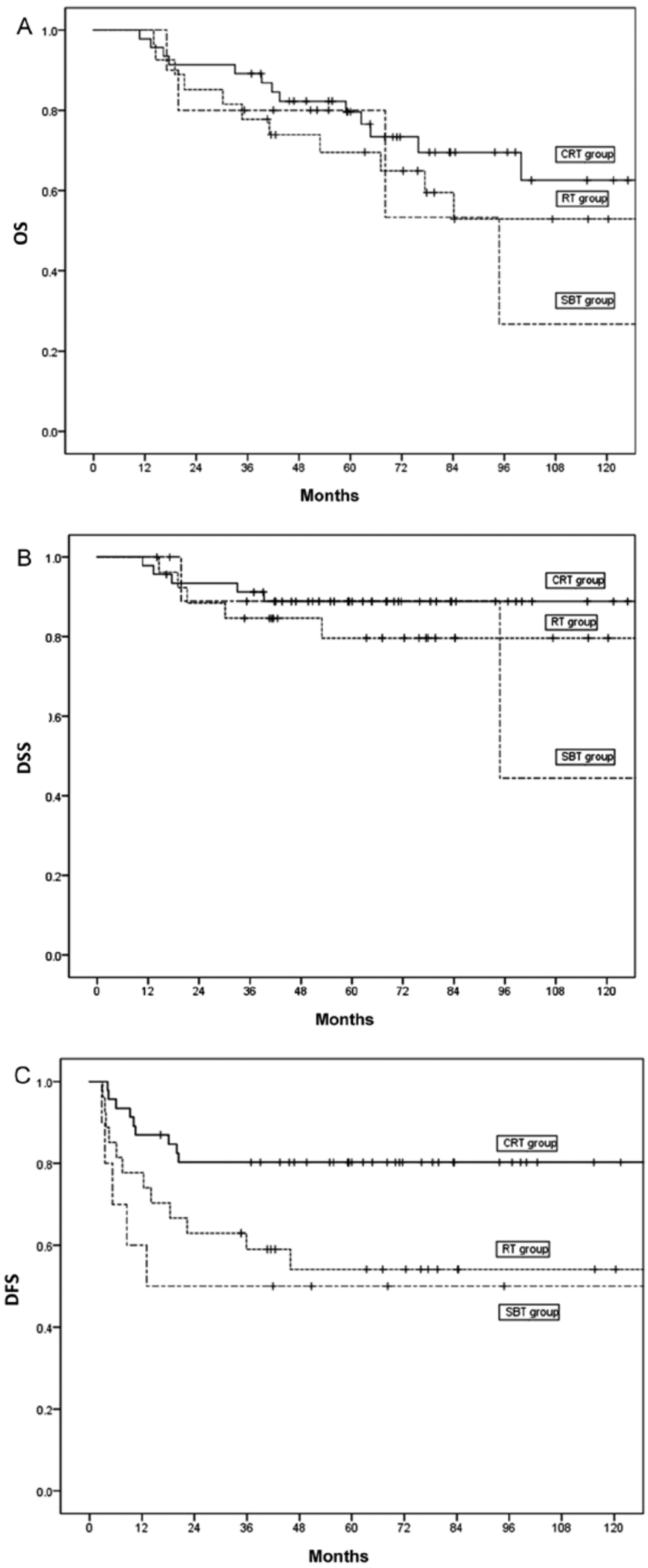

Figure 2. Survival rates between treatment groups. Comparison of (A) OS, (B) DSS and (C) DFS by treatment method of the 83 patients. OS and DSS did not differ significantly among the treatment groups $(\mathrm{P}>0.05)$. However, DFS was significantly higher in the CRT group compared with the RT and SBT groups ( $\mathrm{P}=0.02)$. OS, overall survival; DSS, disease-specific survival; DFS, disease-free survival; CRT, chemoradiotherapy; RT, radiation therapy; SBT, surgery-based treatment. 
Table III. Results of the multivariate analysis of the disease-free survival rate.

\begin{tabular}{|c|c|c|c|}
\hline Parameters & $\begin{array}{l}\text { Hazard ratio } \\
{[\operatorname{Exp}(B)]}\end{array}$ & $95 \% \mathrm{CI}$ & P-value \\
\hline Age & 0.99 & $0.94-1.04$ & 0.72 \\
\hline Primary subsite & & & 0.87 \\
\hline Glottis & 1.0 (ref) & & \\
\hline Supraglottis & 1.19 & $0.43-3.30$ & 0.72 \\
\hline Subglottis & 1.65 & $0.18-15.2$ & 0.66 \\
\hline RT dose & 0.99 & $0.99-1.00$ & 0.41 \\
\hline Treatment method & & & 0.01 \\
\hline CRT & 1.0 (ref) & & \\
\hline RT & 3.8 & $1.35-10.75$ & 0.01 \\
\hline SBT & 6.7 & $1.56-29.39$ & 0.01 \\
\hline
\end{tabular}

Ref, reference; RT, radiation therapy; CRT, chemoradiotherapy; SBT, surgery-based treatment; $\operatorname{Exp}(\mathrm{B})$, exponentiation of the B coefficient.

In the CRT group, neutropenia and dermatitis were the most common findings according to the toxicity criteria. Unlike the RT group, which had distinctive neutropenia, the CRT group demonstrated various toxicities resulting in abnormal hematological findings, mostly neutropenia, anemia and leukopenia. Moreover, the CRT group had higher toxicity in non-hematologic categories. Compared with the RT group, the CRT group also demonstrated significantly more symptoms, such as nausea and diarrhea. However, dermatitis and mucositis were commonly found in the CRT group as in the RT group. In total, eight patients in the CRT group were categorized as having grade IV toxicity. However, one patient delayed the CRT for 2 months due to severe thrombocytopenia; after recovering, the patient completed the remaining RT treatments. It was also identified that no treatment-associated mortalities occurred in the CRT group.

\section{Discussion}

The larynx is an essential organ for speech and swallowing that greatly impacts the quality of life (20). Thus, the goal in the treatment of laryngeal cancer is to treat the disease, while preserving laryngeal function and quality of life (3). However, the requirement to preserve laryngeal function further complicates the decision of treatment methods for laryngeal cancer (21). Moreover, the treatment decision process for patients with laryngeal cancer has to consider numerous factors, such as cancer extent, lung function, the occupation of the patients, lifestyle requirements and psychological support (21). Early laryngeal cancer (T1NOM0 and T2NOM0) is characterized by a low-volume tumor and a low incidence of nodal metastasis; thus, the effective local control of primary treatment is important for laryngeal preservation (5).

There are several guidelines for laryngeal cancer by the NCCN, American Society of Clinical Oncology and United Kingdom National Multidisciplinary Specialty Associations, which recommend that a single treatment modality, including
Table IV. Toxicity occurring in RT and CRT groups was graded according to National Cancer Institution Common Toxicity Criteria version 5.0.

\begin{tabular}{|c|c|c|c|c|c|}
\hline Grade & $\mathrm{I}$ & II & III & IV & Total \\
\hline \multicolumn{6}{|l|}{ RT group (n) } \\
\hline \multicolumn{6}{|l|}{ Hematologic } \\
\hline Anemia & 5 & 2 & 1 & 0 & 8 \\
\hline Leukopenia & 1 & 1 & 0 & 0 & 2 \\
\hline Neutropenia & 19 & 1 & 0 & 0 & 20 \\
\hline Thrombocytopenia & 1 & 0 & 0 & 0 & 1 \\
\hline \multicolumn{6}{|l|}{ Hepatic } \\
\hline LFT & 3 & 0 & 0 & 0 & 3 \\
\hline \multicolumn{5}{|l|}{ Renal } & $\mathrm{Cr}$ \\
\hline Non-Hematologic & 4 & 1 & 0 & 0 & 5 \\
\hline Nausea & 0 & 0 & 1 & 0 & 1 \\
\hline Diarrhea & 1 & 0 & 0 & 0 & 1 \\
\hline Fever & 0 & 0 & 0 & 0 & 0 \\
\hline Dermatitis & 23 & 2 & 1 & 0 & 26 \\
\hline Mucositis & 8 & 9 & 1 & 0 & 18 \\
\hline \multicolumn{6}{|l|}{ CRT group (n) } \\
\hline \multicolumn{6}{|l|}{ Hematologic } \\
\hline Anemia & 23 & 15 & 1 & 0 & 39 \\
\hline Leukopenia & 15 & 15 & 6 & 2 & 38 \\
\hline Neutropenia & 17 & 15 & 6 & 4 & 42 \\
\hline Thrombocytopenia & 16 & 1 & 3 & 1 & 21 \\
\hline \multicolumn{6}{|l|}{ Hepatic } \\
\hline LFT & 4 & 2 & 1 & 0 & 7 \\
\hline $\begin{array}{l}\text { Renal } \\
\mathrm{Cr}\end{array}$ & & & & & \\
\hline Non-Hematologic & 13 & 5 & 0 & 0 & 18 \\
\hline Nausea & 10 & 3 & 1 & 0 & 14 \\
\hline Diarrhea & 6 & 0 & 0 & 0 & 6 \\
\hline Fever & 3 & 0 & 0 & 0 & 3 \\
\hline Dermatitis & 34 & 9 & 0 & 0 & 43 \\
\hline Mucositis & 22 & 11 & 2 & 1 & 36 \\
\hline
\end{tabular}

RT, radiation therapy; CRT, chemoradiotherapy; LFT, liver function test; $\mathrm{Cr}$, creatinine.

larynx-preserving surgery or RT alone, for early laryngeal cancer are accepted treatment options $(4,21,24)$. However, treatment outcomes of T2N0M0 laryngeal cancer are distinctively worse compared with those of T1NOM0 laryngeal cancer, despite both stages being considered the early stage $(24,25)$. Patients with T2N0M0 in particular have a $25-50 \%$ recurrence rate, which is $\geq 2$ times higher compared with that of patients with T1N0M0, due to local control failure in laryngeal cancer (25). Previous studies have identified consistent insufficient treatment outcomes in terms of survival rate, such as laryngeal-preserving survival rate, OS and DSS, in T2NOM0 laryngeal cancer $(10,11)$. Therefore, the present study focused 

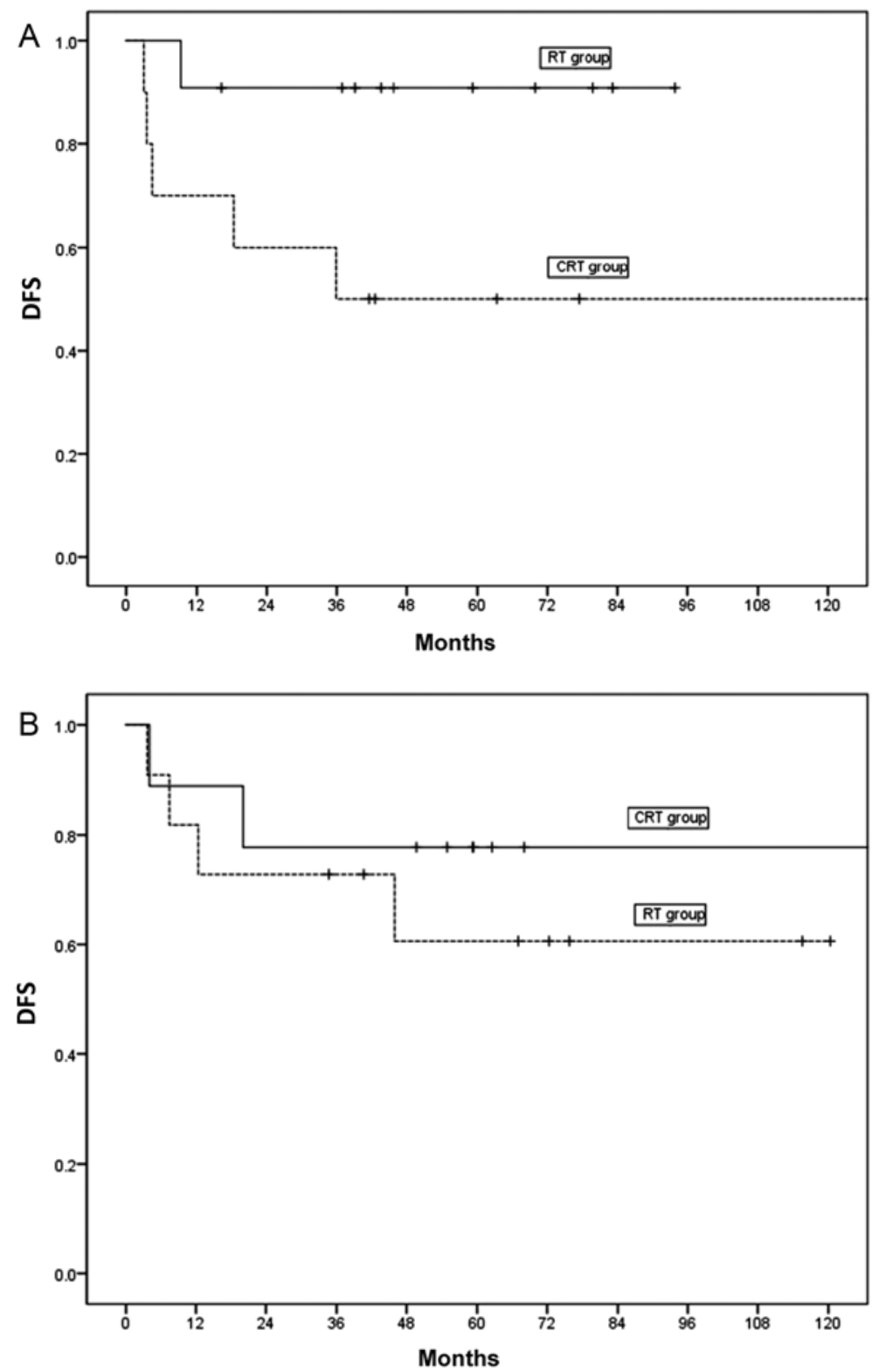

Figure 3. Comparison of DFS in 43 patients with glottic cancer. (A) In 21 patients with glottic cancer and subglottic invasion, DFS was significantly higher in the CRT group compared with the RT group $(\mathrm{P}=0.045)$. (B) In 20 patients with glottis cancer, but no subglottic invasion, DFS did not differ significantly between the two groups $(\mathrm{P}=0.47)$. DFS, disease-free survival; $\mathrm{CRT}$, chemoradiotherapy; RT, radiation therapy.

on comparing long-term treatment outcomes according to treatment method in patients with T2N0M0 laryngeal cancer. Furthermore, patients in this study were divided into RT, CRT and SBT groups by their initial treatment method. However, there were only ten patients in the SBT group, which is insufficient to enable assessment of the treatment outcomes of the SBT group, and thus is a limitation of the study; although the comparison between the RT and CRT groups remains important.

In this study, the CRT group had a significantly higher local control rate $(87.0$ vs. $55.6 \%)$, lower recurrence rate (19.6 vs. 44.4\%) and higher DFS (80 vs. 55\% in 5 years) compared with the RT group. The multivariate analysis results also suggested that the treatment method was the most significant factor for DFS, in that the CRT group had a superior prognosis compared with the RT and SBT groups. However, there was no significant intergroup difference in
OS or DSS, as improved salvage treatment after recurrence could dilute the adverse effects of recurrence on OS and DSS. Akimoto et al (7) reported that voice preservation and DFS in patients treated with CRT are significantly superior compared with those of RT alone in T2N0M0 laryngeal cancer. Previous studies by Furusaka et al (12) and Nishimura et al (13) also revealed that CRT improves laryngeal preservation compared with RT alone in T2N0M0 laryngeal cancer. Thus, the results of these previous studies are consistent with those of the present study. In laryngeal cancer, local control is directly associated with laryngeal preservation (14). With the development of the larynx-preserving surgery technique, larynx-preserving surgery is possible without total laryngectomy, even in patients with recurrent laryngeal cancer after RT (14). However, in these cases, due to the serious quality of life deterioration, such as chronic aspiration, the improvement in local control is essential for improving functional laryngeal preservation (17). 
Thus, in T2N0M0 laryngeal cancer, CRT may be an alternative method that improves local control and, in turn, improves laryngeal preservation and DFS.

It was hypothesized that the poor local control rate of the RT group may be due to understaging. With regards to the present study, in the clinical staging system, based on CT and laryngoscopy findings, some patients classified as having T2N0M0 laryngeal cancer may actually have minor paraglottic and pre-epiglottic space invasion (T3), or minor thyroid cartilage invasion (T4a), which are not prominent on a CT scan. Moreover, these patients with deep tissue invasion could experience a difference in local control between the RT and CRT groups; however, there is no method to filter out these patients in the clinical setting. The present study reviewed the CT scans of all enrolled patients, but no classifications were changed from T2N0M0 to T3 or T4a. In particular, most patients with T2N0M0 glottic cancer had AC invasion, which can cause minor invasion of the thyroid cartilage as there is no perichondrium at the insertion of Broyles' ligament, making it difficult to identify on a CT scan (26). The present results suggested that $81.4 \%$ of the patients had AC invasion, while 53.5\% had AC invasion and subglottic invasion, which could be assumed due to deep tissue invasion of the cancer. The present results supported the above hypothesis, as for patients with AC and subglottic invasion, the CRT group had significantly improved DFS compared with the RT group; while among patients without subglottic invasion, there was no statistically significant intergroup difference.

Toxicity is an issue that is associated with chemotherapy (27). In the present study, more patients in the CRT group compared with the RT group showed toxicity. In particular, the proportion of patients with a toxicity higher than grade III was increased in the CRT group. However, there were no cases of mortality due to toxicity, and all cases of treatment-associated toxicity were manageable. Furthermore, the improvement of various drugs and the ability of infection control to manage acute toxicity of chemotherapy, including the severe neutropenia and associated fever, significantly contributed to the low mortality and morbidity of chemotherapy in the present study.

The main limitation of the current study is that the sample size of case series was not sufficient for an accurate multivariate analysis. This study was conducted in a tertiary referral hospital with a regional cancer center in South Korea, but the number of patients with T2N0M0 laryngeal cancer did not approach 100 cases during the 10 years. Therefore, further studies with larger focused cohorts are required to assess the applicability of the present results, and a multicenter randomized trial may be an alternative. However, the present study provided a basis for conducting a randomized trial to improve treatment outcome for T2N0M0 laryngeal cancer.

In conclusion, while this retrospective study is insufficient to draw definitive conclusions, the present results indicated that CRT had a positive impact on local control and DFS with manageable toxicity in T2NOM0 laryngeal cancer. Thus, a randomized prospective trial should be performed to compare RT, CRT and SBT for treating T2N0M0 laryngeal cancer.

\section{Acknowledgements}

The authors would like to thank Dr SS Kweon (Chonnam National University) for the consultation of statistics.

\section{Funding}

This study was supported by the Chonnam National University Hwasun Hospital Institute for Biomedical Science (Hwasun, South Korea; grant no. HCRI 16922-22) and the National Research Foundation of Korea (Daejeon, South Korea; grant no. 2017R1C1B1009843).

\section{Availability of data and materials}

The datasets used and/or analyzed during the current study are available from the corresponding author upon reasonable request.

\section{Authors' contributions}

TMY and SCL designed the present study EKJ, JJ and TMY analyzed the data and drafted the initial manuscript. SJ, JK, DHL and JKL acquired and analyzed the data. WC, HKK, JH, HS, WB, SC and IC contributed to the interpretation of data. All authors read and approved the final manuscript.

\section{Ethics approval and consent to participate}

This study was approved by the Ethics Committee of the Institutional Review Board of Chonnam National University Hwasun Hospital (Hwasun, South Korea; approval no. CNUHH-2019-178) under the exemption of patients informed consent. The Ethics Committee of the Institutional Review Board allows the exemption of patients informed consent for retrospective studies, as a retrospective study has a extremely low risk of influencing subjects, and obtaining consent is very difficult in the retrospective studies.

\section{Patient consent for publication}

Not applicable.

\section{Competing interests}

The authors declare that they have no competing interests.

\section{References}

1. Siegel R, Naishadham D and Jemal A: Cancer statistics, 2013. CA Cancer J Clin 63: 11-30, 2013.

2. Groome PA, O'Sullivan B, Irish JC, Rothwell DM, Schulze K, Warde PR, Schneider KM, Mackenzie RG, Hodson DI, Hammond JA, et al: Management and outcome differences in supraglottic cancer between Ontario, Canada, and the Surveillance, Epidemiology, and End Results areas of the United States. J Clin Oncol 21: 496-505, 2003.

3. Mendenhall WM, Werning JW, Hinerman RW, Amdur RJ and Villaret DB: Management of T1-T2 Glottic carcinomas. Cancer 100: 1786-1792, 2004.

4. Pfister DG, Spencer S, Adelstein D, Adkins D, Brizel DM, Burtness B, Busse PM, Caudell JJ, Cmelak AJ, Colevas AD, et al (eds): National Comprehensive Cancer Network: NCCN Clinical Practice Guidelines in Oncology (NCCN Guidelines) Head and Neck Cancers (Version 1), 2020. www.nccn.org. Accessed April 07, 2020.

5. Feng Y, Wang B and Wen S: Laser surgery versus radiotherapy for T1-T2N0 glottic cancer: A meta-analysis. ORL J Otorhinolaryngol Relat Spec 73: 336-342, 2011. 
6. Yoo J, Lacchetti C, Hammond JA and Gilbert RW; Head and Neck Cancer Disease Site Group: Role of endolaryngeal surgery (with or without laser) versus radiotherapy in the management of early (T1) glottic cancer: A systematic review. Head Neck 36: $1807-1819,2014$.

7. Akimoto T, Nonaka T, Kitamoto $\mathrm{Y}$, Ishikawa $\mathrm{H}$, Ninomiya $\mathrm{H}$, Chikamatsu K, Furuya N, Hayakawa K, Mitsuhashi N and Nakano T: Radiation therapy for T2N0 laryngeal cancer: A retrospective analysis for the impact of concurrent chemotherapy on local control. Int J Radiat Oncol Biol Phys 64: 995-1001, 2006

8. Laccourreye O, Weinstein G, Brasnu D, Trotoux J and Laccourreye H: Vertical partial laryngectomy: A critical analysis of local recurrence. Ann Otol Rhinol Laryngol 100: 68-71, 1991.

9. Brumund KT, Gutierrez-Fonseca R, Garcia D, Babin E, Hans S and Laccourreye O: Frontolateral vertical partial laryngectomy without tracheotomy for invasive squamous cell carcinoma of the true vocal cord: A 25-year experience. Ann Otol Rhinol Laryngol 114: 314-322, 2005.

10. Furusaka T, Matuda H, Saito T, Katsura Y and Ikeda M: Long-term observations and salvage operations on patients with T2N0M0 squamous cell carcinoma of the glottic larynx treated with radiation therapy alone. Acta Otolaryngol 132: 546-551, 2012.

11. Arshad H, Jayaprakash V, Gupta V, Cohan DM, Ambujakshan D, Rigual NR, Singh AK and Hicks WL Jr: Survival differences between organ preservation surgery and definitive radiotherapy in early supraglottic squamous cell carcinoma. Otolaryngol Head Neck Surg 150: 237-244, 2014.

12. Furusaka T, Matsuda A, Saito T, Katsura Y and Ikeda M Concurrent chemoradiation therapy with docetaxel (DOC) for laryngeal preservation in T2NOM0 glottic squamous cell carcinomas. Acta Otolaryngol 133: 99-112, 2013.

13. Nishimura G, Tsukuda M, Mikami Y, Matsuda H, Horiuchi C, Taguchi T, Takahashi M, Kawakami M, Watanabe M, Niho T, et al: Efficacy of concurrent chemoradiotherapy for $\mathrm{T} 1$ and $\mathrm{T} 2$ laryngeal squamous cell carcinoma regarding organ preservation. Anticancer Res 29: 661-666, 2009.

14. American Society of Clinical Oncology, Pfister DG, Laurie SA, Weinstein GS, Mendenhall WM, Adelstein DJ, Ang KK, Clayman GL, Fisher SG, Forastiere AA, et al: American society of clinical oncology clinical practice guideline for the use of larynx-preservation strategies in the treatment of laryngeal cancer. J Clin Oncol 24: 3693-3704, 2006.

15. Urba S, Wolf G, Eisbruch A, Worden F, Lee J, Bradford C, Teknos T, Chepeha D, Prince M, Hogikyan N and Taylor J: Single-cycle induction chemotherapy selects patients with advanced laryngeal cancer for combined chemoradiation: A new treatment paradigm. J Clin Oncol 24: 593-598, 2006.

16. Lefebvre JL: Laryngeal preservation in head and neck cancer: Multidisciplinary approach. Lancet Oncol 7: 747-755, 2006.
17. Forastiere AA, Zhang Q, Weber RS, Maor MH, Goepfert H, Pajak TF, Morrison W, Glisson B, Trotti A, Ridge JA, et al: Long-term results of RTOG 91-11: A comparison of three nonsurgical treatment strategies to preserve the larynx in patients with locally advanced larynx cancer. J Clin Oncol 31: 845-852, 2013

18. Pignon JP, le Maître A, Maillard E and Bourhis J; MACH-NC Collaborative Group: Meta-analysis of chemotherapy in head and neck cancer (MACH-NC): An update on 93 randomised trials and 17,346 patients. Radiother Oncol 92: 4-14, 2009.

19. Amin MB, Edge S, Greene F, Byrd DR, Brookland RK, Washington MK, Gershenwald JE, Compton CC, Hess KR, Sullivan DC, et al (eds): AJCC Cancer Staging Manual. 8th edition. Springer, New York, NY, 2017.

20. Korean Society of Thyroid-Head and Neck Surgery Guideline Task Force, Ahn SH, Hong HJ, Kwon SY, Kwon KH, Roh JL, Ryu J, Park JH, Baek SK, Lee GH, et al: Guidelines for the surgical management of laryngeal cancer: Korean society of thyroid-head and neck surgery. Clin Exp Otorhinolaryngol 10: $1-43,2017$.

21. Forastiere AA, Ismaila N, Lewin JS, Nathan CA, Adelstein DJ, Eisbruch A, Fass G, Fisher SG, Laurie SA, Le QT, et al: Use of Larynx-Preservation strategies in the treatment of laryngeal cancer: American society of clinical oncology clinical practice guideline update. J Clin Oncol 36: 1143-1169, 2018.

22. Shanei A, Abedi I, Saadatmand P, Amouheidari AR and Akbari-Zadeh H: Comparison of 3D conformal and intensity modulated radiotherapy in early stage oral tongue cancer: Dosimetric and radiobiological evaluation. Int J Radiat Res 18: 33-42, 2020.

23. US Department of Health and Human Services; National Institutes of Health; National Cancer Institute: Common Terminology Criteria For Adverse Events (CTCAE), Version 5.0. November 27, 2017.

24. Jones TM, De M, Foran B, Harrington K and Mortimore S: Laryngeal cancer: United Kingdom National Multidisciplinary guidelines. J Laryngol Otol 130: S75-S82, 2016.

25. Hirasawa N, Itoh Y, Ishihara S, Kubota S, Itoh J, Fujimoto $Y$, Nakashima $T$ and Naganawa $S$ : Radiotherapy with or without chemotherapy for patients with T1-T2 glottic carcinoma: Retrospective analysis. Head Neck Oncol 2: 20, 2010.

26. Mor $\mathrm{N}$ and Blitzer A: Functional anatomy and oncologic barriers of the larynx. Otolaryngol Clin North Am 48: 533-545, 2015.

27. Culakova E, Thota R, Poniewierski MS, Kuderer NM, Wogu AF, Dale DC, Crawford J and Lyman GH: Patterns of chemotherapy-associated toxicity and supportive care in US oncology practice: A nationwide prospective cohort study. Cancer Med 3: 434-444, 2014 